\title{
Preprint Server
}

Umar Ahmad

Potential competing interests: The author(s) declared that no potential competing interests exist.

A computer server where scientists and researchers post manuscripts before peer review by the journals 